\title{
Comprehensive study on physical, elastic and shielding properties of lead zinc phosphate glasses
}

\begin{abstract}
A series of ternary phosphate glasses in the form of $(\mathrm{PbO}) \mathrm{x}(\mathrm{ZnO}) 60-\mathrm{x}(\mathrm{P} 2 \mathrm{O} 5) 40$ where $\mathrm{x}=0$ $60 \mathrm{~mol} \%$, have been successfully prepared by conventional melt-quenching technique. The physical and elastic properties of the glasses have been investigated using pulse echo technique. The longitudinal and shear velocity of the glasses were measured using the MBS8000 ultrasonic data acquisition system at $10 \mathrm{MHz}$ frequency in room temperature. The density, ultrasonic velocity and elastic moduli are found to be composition dependent and the correlation between the elastic moduli with the atomic packing density is discussed in detailed. The shielding parameters, mass attenuation coefficients, half value layers and exposure buildup factor (EBF) values have been computed using WinXCom program with the use of GP fitting method, and variation of shielding parameters are discussed for the effect of $\mathrm{PbO}$ addition into the glasses and photon energy. An increase in the density of the glasses results in a change in crosslink density. The sound velocity and elastic properties increased with $\mathrm{PbO}$ content and increase in Poisson's ratio trend suggests that the rigidity of the glasses has decreased. Besides, the replacement of $\mathrm{ZnO}$ by $\mathrm{PbO}$ causes an increase in mass attenuation coefficient, while the half value layer and the exposure buildup factor were decreased and these glasses has been potentially used as shielding material.
\end{abstract}

Keyword: Lead zinc phosphate glasses; Ultrasonic velocity; Elastic moduli; Shielding parameter; Mass attenuation coefficient 